Bangladesh J. Plant Taxon. 27(1): 85-101, 2020 (June)

(C) 2020 Bangladesh Association of Plant Taxonomists

\title{
AN ANNOTATED CHECKLIST OF WEED FLORA IN ODISHA, INDIA
}

\author{
Taranisen Panda*, Nirlipta Mishra ${ }^{1}$, Shaikh Rahimuddin ${ }^{1}$, \\ Bikram K. Pradhan and Raj B. Mohanty ${ }^{2}$ \\ Department of Botany, Chandbali College, Chandbali, Bhadrak-756133, Odisha, India
}

Keywords: Bhadrak district; Diversity; Ecosystem services; Traditional medicines; Weed.

\begin{abstract}
This study consolidated our understanding on the weeds of Bhadrak district, Odisha, India based on both bibliographic sources and field studies. A total of 277 species of weed taxa belonging to 198 genera and 65 families are reported from the study area. About $95.7 \%$ of these weed taxa are distributed across six major superorders; the Lamids and Malvids constitute $43.3 \%$ with 60 species each, followed by Commenilids (56 species), Fabids (48 species), Companulids (23 species) and Monocots (18 species). Asteraceae, Poaceae, and Fabaceae are best represented. Forbs are the most represented $(50.5 \%)$, followed by shrubs $(15.2 \%)$, climber $(11.2 \%)$, grasses $(10.8 \%)$, sedges $(6.5 \%)$ and legumes (5.8\%). Annuals comprised about 57.5\% and the remaining are perennials. As per Raunkiaer classification, the therophytes is the most dominant class with 135 plant species $(48.7 \%)$. The use of weed for different purposes as indicated by local people is also discussed. This study provides a comprehensive and updated checklist of the weed speciesof Bhadrak district which will serve as a tool for conservation of the local biodiversity.
\end{abstract}

\section{Introduction}

India, a country with heterogeneous landforms, shows great variation from one region to another in respect of climate, altitude and vegetation.The country has 60 agroeco-subregions and each agro-eco-subregion has been divided into agro-eco-units at the district level for developing long term land use strategies (Gajbhiye and Mandal, 2006). Climatic, edaphic and biotic factors prevailing in each of the agro-ecological regions influence the formation of vegetation of that area (Rao et al., 2014). The diversity of native flora is an important component of ecosystems that has a primary role in protecting the environmental stability of a region (Cunningham et al., 2015). DeWet and Harlan (1975) classified plants of nature into three categories: i) wild plants (which grow naturally outside the human disturbed habitat), ii) domesticates/ crops (which are artificially propagated and often require cultivation and care by humans in order to grow and make use of environmental resources), and iii) weeds (which thrive in habitats that are continuously disturbed by humans).

The terms weed, invader and colonizer have often been used in a conflicting way. The distinctions between them are quite subtle and result from differing viewpoints. According to Rejmanek (1995), weeds interfere with human land use; colonizers are successful at establishing following disturbance; and invaders are species introduced into their non-native habitat. There is substantial overlap among these terms. A plant may be considered as only one of these, or it may be included in all of thesecategories. Many varying definitions have been developed for weeds, depending on each particular situation where they occur and the plants involved. Thomas et al.

*Corresponding author. email: taranisenpanda@yahoo.co.in

${ }^{1}$ Department of Zoology, Chandbali College, Chandbali, Bhadrak- 756133, Odisha, India.

${ }^{2}$ Ex-Reader in Botany, Plot No. 1311/7628, Satya Bihar, Rasulgarh, Bhubaneswar-751010, Odisha, India. 
(2002) stated that a weed as a plant growing where it is not desired, or a plant out of place. According to Barbara et al. (2003), a weed is a native or introduced (alien) species that has a perceived negative ecological or economic effect on agricultural or natural systems. Navas (1991) defined a weed as "a plant that form populations that are able to enter cultivated habitats,markedly disturbed or occupied by man, and potentially depress or displace the resident plant populations which are deliberately cultivated or are of ecological and/or aesthetic interest".

A weed is only a weed under specific circumstances, that the inclusion of a plant into this category is arbitrarily based on human perceptions and that a specific plant species will not always be considered a weed (Crawley, 1997b).Weeds have some pinpointing characters, such as short seed dormancy, high seed germination rate, environmental plasticity, high seedling growth and reproductive capacity, short life cycle, self-compatibility, efficient and well organized methods of seed dispersal, allelopathy and tolerance to abiotic and biotic stresses (El-Sheikh, 2013a).Furthermore, weeds are able to survive and grow in different ecological habitats, with their occurrence being very sensitive to changes in agricultural practices (Fried et al., 2010). Due to these reason weeds are becoming dominant all over the world (Holm et al., 1997). Moreover, the current climate change and natural calamities (cyclones, storms and associated floods) have emerged as the greatest ecological challenge of the 21st century which will affect prevalence of weed species, their distribution and dispersal to invade new ecosystems (Kang and Banga, 2013; Singh et al., 2011). In addition, the effect of human mediated processes on weed species composition cannot be overlooked (Hyvonen, 2007).Those plants which are susceptible to a particular kind of disturbance decrease in number or even disappear (Bergmeier, 2006).

Negative connotations are commonly invoked by the term weed, when referring to biodiversity assets (Martin-Fores et al., 2017).Indeed, since the beginning of crop production weeds have represented a serious constraint to worldwide agriculture, as when left uncontrolled they can cause severe economic losses by reducing detrimentally crop yield and quality (Travlos $e t$ al., 2018).Weed growth represents a major source of inefficiency, diverting scarce resources (nutrients, water, sunlight and labour) for cultivated crops (Nyarko and Datta, 1993).They can significantly influence crop disease incidence by acting as vectors or reservoirs of plant pathogens (Wisler and Norris, 2005). Weeds infestation also slow down harvesting operation, increase the cost of production, decrease excellence of fertile lands and germination capability of crops seed due to the phytotoxins or allelochemicals and reduce the market value of crops (Algandaby and Salama, 2016).

However, the benefits of weeds are less well understood.Weeds may increase crop growth under certain circumstances. For example, in some dry areas of India, three 'weeds' (Arnebia hispidissima (Lehm.) DC., Borreria articularis (L.f.) Williams and Celosia argentea) increase the growth of millet (bajra, Pennisetum typhoideum) (Bhandari and Sen, 1979). Weeds form the basis of the agro-ecosystem food web and provide various ecosystem services, such as provisioning of food, medicine, prevention of soil erosion, and livestock feed (Bastiaans et al. 2000; Yamaguchi and Umemoto, 1996). Therefore, it is of a vital importance for every country to keep a record of the diversity and distribution of its weeds, and identify whether they are native or exotic/introduced/ aliens/ invader. Potentially serious new weeds are often overlooked until they are widely naturalized and having harmful impact on agricultural production and environment (Waterhouse, 2003).Thus, there is an urgent need to carry out floristic surveys, especially in regions where the flora is not well documented. Fair amount of studies are encountered to document the weed flora of India (Bhattacharjya and Sarma, 2016; Tiwari et al., 2016; Sinha and Banerjee, 2018). However, very little is known about weed flora of Odisha (Mallick et al., 2015).To date, published information is not available on weed plant communities of Bhadrak district of Odisha, India. The main aim of the presented research is to provide the baseline 
information on composition and distribution of weed communities prevailing in the Bhadrak region.

\section{Materials and Methods}

Study area

Odisha is the ninth largest state of India by area and the eleventh largest by population. It is located in the east coast of India $\left(17.48^{\circ}-22.34^{\circ} \mathrm{N}\right.$ and $\left.81.24^{\circ}-87.29^{\circ} \mathrm{E}\right)$ with the Bay of Bengal forming its eastern and south eastern frontiers. The entire territory lies in the tropical zone as a result of which high temperature is recorded particularly during April-May. However, the sea exercises a moderating influence over the climate of the coastal belt whereas the hill tracts experience an extreme climate.The forest found in this region is tropical moist deciduous (Champion and Seth, 1968).

Bhadrak district $\left(20^{\circ} 43^{\prime}-21^{\circ} 13^{\prime} \mathrm{N}\right.$ and $\left.86^{\circ} 6^{\prime}-87^{\circ} \mathrm{E}\right)$ is located in Northeast Odisha. It spreads over $2505 \mathrm{~km}^{2}$ having 1.507 million inhabitants (2011 Census). Four other districts namely Balasore, Kendrapara, Jajpur and Koenjher surround Bhadrak district while a part is bounded by the Bay of Bengal. Three distinct annual seasons are the rainy (mid June to mid October), winter (mid October to February) and summer (March to mid June) season. The maximum and minimum temperatures range from $37.4^{\circ} \mathrm{C}$ to $17.7^{\circ} \mathrm{C}$ respectively and the annual average rainfall is approximately $1428 \mathrm{~mm}$.It is characterized by periodic earth tremors, thunder storms in the rains and dust storms in April and May. The district covers about $1.61 \%$ of the total land area of the state and contributes $3.59 \%$ of the state's population. About $86.66 \%$ of the inhabitants are villagers and the people are engaged in agricultural practices as their primary occupation.

\section{Data collection}

Extensive field surveys (June 2015- May 2018) were carried out to document and enlist the weed flora in diverse habitats following established and standard procedures (Martin, 1995). The information on the weed plants was obtained through questionnaires, complemented by free interviews and informal conversations (Martin, 1995). The information regarding the weed species has been gathered mostly from local farmers, elderly and knowledgeable persons. Personal interviews and group discussions carried out in the local language revealed specific information about the plants, which were further compared and authenticated by crosschecking (Cunningham, 2001).During field study, the economic uses of these species if any were discussed with the local people. Weeds were identified with available regional floras (Saxena and Brahmam 1996).The plant species are enumerated and arranged as per Angiosperm Phylogeny Group III Classification (APG III, 2009). The lifeforms of plant species were recognized through Raunkiaer (1934) classification. The voucher specimens were deposited in the herbarium of the Department of Botany, Chandbali College, Chandbali. The weed plants were represented alphabetically according to their scientific names, local name if any, family, habit, life span, life form, and uses.

\section{Results and Discussion}

Two hundred and seventy seven species belonging to 198 genera of 65 flowering plant families were recorded in the study area, representing ten superorders and thirty orders as per APG III classification (Table 1). Among the reported plants, $43.3 \%$ of the taxa were recorded from the superorder Lamids and Malvids (each with 60 species), 20.2\% from superorder commelinids, $17.3 \%$ from Fabids, and 8.3\% from superorder Companulids. Order Poales, Carylophyllales, Lamiales, Sonales, Asterales, Fabales, Malvales and Malphigiales accounted for about $75 \%$ of the species in the district. Twenty seven families are only represented by one species; examples include Capparaceae, Molluginaceae, Oxalidaceae, Papaveraceae and Vitaceae.The largest family 
Table 1. List of weed flora recorded from Bhadrak district, arranged according to the Angiosperm Phylogeny Group Classification III.

\begin{tabular}{|c|c|c|c|c|c|c|}
\hline Superorder/Order & Family/Species & $\begin{array}{l}\text { Common } \\
\text { name }\end{array}$ & Habit & $\begin{array}{l}\text { Life } \\
\text { span }\end{array}$ & $\begin{array}{l}\text { Life } \\
\text { form }\end{array}$ & Uses \\
\hline \multicolumn{7}{|c|}{ EARLY ANGIOSPERMS } \\
\hline \multirow[t]{4}{*}{ Nymphaeales } & Nymphaeaceae & & & & & \\
\hline & Nymphaea nouchali Burm. f. & Kain & Forb & $\mathrm{P}$ & Hyd & $\mathrm{M}, \mathrm{E}$ \\
\hline & Nymphaea pubescens Willd. & Rangakain & Forb & $\mathrm{P}$ & Hyd & $\mathrm{E}$ \\
\hline & Euryale ferox Salisb. & Kanta Padma & Forb & $\mathrm{P}$ & Hyd & M \\
\hline \multicolumn{7}{|l|}{ MAGNOLIIDS } \\
\hline \multirow[t]{3}{*}{ Piperales } & Aristolachiaceae & & & & & \\
\hline & $\begin{array}{l}\text { Aristolochia indica } \mathrm{L} . \\
\text { Piperaceae }\end{array}$ & Balbolena & Climber & $\mathrm{p}$ & Cry & M \\
\hline & Peperomia pellucida (L.) Kunth & & Forb & A & Hem & M \\
\hline \multirow[t]{2}{*}{ Laurales } & Lauraceae & & & & & \\
\hline & Cassytha filiformis $\mathrm{L}$. & Nirmuli & Climber & $\mathrm{P}$ & $\mathrm{Ph}$ & M \\
\hline \multicolumn{7}{|l|}{ MONOCOTS } \\
\hline \multirow[t]{14}{*}{ Alismatales } & Alismataceae & & & & & \\
\hline & $\begin{array}{l}\text { Sagittaria sagittifolia } \mathrm{L} \text {. } \\
\text { Aponogetonaceae }\end{array}$ & & Forb & $\mathrm{P}$ & Hyd & $\mathrm{E}$ \\
\hline & $\begin{array}{l}\text { Aponogeton natans (L.) Engl. \& } \\
\text { Krause }\end{array}$ & Jhechu & Forb & $\mathrm{P}$ & Hyd & $\mathrm{E}$ \\
\hline & $\begin{array}{l}\text { Aponogeton undulatus Roxb. } \\
\text { Araceae }\end{array}$ & & Forb & $\mathrm{P}$ & Hyd & $\mathrm{E}$ \\
\hline & Alocasia macrorrhizos (L.) G.Don. & Badasaru & Forb & $\mathrm{P}$ & Cry & $\mathrm{E}$ \\
\hline & $\begin{array}{l}\text { Amorphophallus paeoniifolius } \\
\text { (Dennst.)Nicolson }\end{array}$ & Olua & Forb & $\mathrm{P}$ & Cry & $\mathrm{E}$ \\
\hline & Caladium bicolor (Aiton) Vent. & & Forb & A & Cry & NK \\
\hline & Colocasia esculenta (L.) Schott. & Saru & Forb & A & Hyd & $\mathrm{M}, \mathrm{E}$ \\
\hline & $\begin{array}{l}\text { Pistia stratiotes } \mathrm{L} . \\
\text { Hydrocharitaceae }\end{array}$ & Borajhanji & Forb & $P$ & Hyd & M \\
\hline & Hydrilla verticillata (L. f.) Royle. & Chingudiadala & Forb & $P$ & Hyd & M \\
\hline & Ottelia alismoides (L.) Pers. & Panikundri & Forb & $\mathrm{P}$ & Hyd & $\mathrm{M}, \mathrm{E}$ \\
\hline & Vallisneria natans (Lour.) Hara & & Forb & A & Hyd & E \\
\hline & Lemnaceae & & & & & \\
\hline & $\begin{array}{l}\text { Wolffia globosa (Roxb.) Hatog.\& } \\
\text { Plas }\end{array}$ & & Forb & A & Hyd & $\mathrm{E}$ \\
\hline \multirow[t]{3}{*}{ Pandanales } & Pandanaceae & & & & & \\
\hline & Pandanus fascicularis Lam. & Kia & Shrub & $\mathrm{P}$ & $\mathrm{Ph}$ & $\mathrm{BF}$ \\
\hline & Pandanus foetidus Roxb. & Lunikia & Shrub & $\mathrm{P}$ & $\mathrm{Ph}$ & Biofencing \\
\hline \multirow[t]{2}{*}{ Liliales } & Colchicaceae & & & & & \\
\hline & Gloriosa superba $\mathrm{L}$. & Ognisikha & Climber & A & $\mathrm{Cr}$ & M \\
\hline \multirow[t]{5}{*}{ Asparagales } & Amaryillidaceae & & & & & \\
\hline & Crinum asiaticum $\mathrm{L}$. & Arsa & Forb & $\mathrm{P}$ & $\mathrm{Cr}$ & $\mathrm{M}, \mathrm{E}$ \\
\hline & Asparagaceae & & & & & $\mathrm{BF}$ \\
\hline & Agave americana $\mathrm{L}$. & Baramasi & Shrub & $\mathrm{P}$ & $\mathrm{Ph}$ & $\mathrm{BF}$ \\
\hline & $\begin{array}{l}\text { Sansevieria roxburghiana Schult. \& } \\
\text { Schult. f. }\end{array}$ & Muruga & Forb & $\mathrm{P}$ & $\mathrm{Ph}$ & \\
\hline
\end{tabular}




\section{COMMELINIDS}

\section{Commelinales Commelinaceae}

Aneilema vaginatum (L.) R.Br.

Commelina benghalensis $\mathrm{L}$.

Commelina difusa Burm.f.

Cyanotis axillaris (L.) Schult. \&

Schult. f.

Murdannia nudiflora (L.) Brenan

Pontederiaceae

Eichhornia crassipes (Mart.)Solms

Monocharia hastata (L.)Solm

Poales

\section{Poaceae}

Brachiaria reptans (L.) Garde. \&

Hubb.

Chloris barbata $\mathrm{Sw}$.

Chrysopogon aciculatus (Retz.) Guguchia
Trin.

Coix lacryma-jobi $\mathrm{L}$.

Cynodon dactylon (L.) Pers.

Dactyloctenium aegyptium (L.)

Willd.

Digitaria cilliaris Retz. Koeler

Echinochloa colona (L.) Link

Echinochloa crusgalli (L.) P. Beauv.

Eleusine indica (L.) Gaertn.

Eragrostis ciliata (Roxb.)Nees

Eragrostis gangetica (Roxb.) Steud.

Heteropogon contortus (L.) P.

Beauv.

Imperata cylindrica (L.) Raeusch.

Isachne globosa (Thunb.) Kuntze

Ischaemum rugosum Salisb.

Leptochloa chinensis (L.) Nees

Oplismenus burmanii (Retz.) P.

Beauv.

Oryza rufipogon Griff.

Panicum psilopodium Trin.

Panicum repens $\mathrm{L}$.

Paspalum distichum $\mathrm{L}$.

Phragmites karka (Retz.) Trin.ex

Steud.

Saccharum spontaneum $\mathrm{L}$.

Setaria intermedia Roem. \& Schult.

Setaria pumila (Poir.) Roem. \&

Schult.

Setaria verticillata (L.) P. Beauv.

Sporobolus indicus (L.) R. Br.

Vetiveria zizanioides (L.) Nash.

Xyridaceae

Xyris indica $\mathrm{L}$.
Kansiri

Kanduli

Bilatidala

Gargara

Duba

Kakhuriya

Swanghas

Dhera

Anamandia

Kankra chare

Dauria

Chhana ghas

Tuli

Bhuru

Kanguria

Balunga

Reda

Noto

Kashatundi

Sial legunda

Kankra chara

Bena

\begin{tabular}{|c|c|c|c|}
\hline Forb & A & Ch & FD \\
\hline Forb & A & Ch & $\mathrm{M}$ \\
\hline Forb & A & $\mathrm{Ch}$ & M \\
\hline Forb & A & $\mathrm{Ch}$ & FD \\
\hline Forb & A & Ch & FD \\
\hline Forb & $\mathrm{P}$ & Hyd & NK \\
\hline Forb & A & Hyd & E \\
\hline Grass & A & $\mathrm{Ch}$ & FD \\
\hline Grass & $\mathrm{P}$ & Hem & FD \\
\hline Grass & $\mathrm{P}$ & $\mathrm{Cr}$ & $\mathrm{M}$ \\
\hline Grass & A & Th & FD \\
\hline Grass & $\mathrm{P}$ & Hem & $\mathrm{M}, \mathrm{R}$ \\
\hline Grass & A & Hem & FD \\
\hline Grass & A & $\mathrm{Ch}$ & FD \\
\hline Grass & A & Th & E \\
\hline Grass & A & Th & E \\
\hline Grass & A & Hem & FD \\
\hline Grass & $\mathrm{P}$ & $\mathrm{Cr}$ & FD \\
\hline Grass & A & $\mathrm{Cr}$ & FD \\
\hline Grass & $\mathrm{P}$ & $\mathrm{Cr}$ & FD \\
\hline Grass & $\mathrm{P}$ & Hem & FD \\
\hline Grass & A & Th & FD \\
\hline Grass & A & Hem & FD \\
\hline Grass & A & Hem & FD \\
\hline Grass & A & Th & FD \\
\hline Grass & $\mathrm{P}$ & Th & FD \\
\hline Grass & A & Th & FD \\
\hline Grass & $\mathrm{P}$ & Th & FD \\
\hline Grass & $\mathrm{P}$ & Th & FD \\
\hline Grass & A & Th & FD \\
\hline Grass & $\mathrm{P}$ & Hel & FD \\
\hline Grass & A & Th & FD \\
\hline Grass & A & Th & FD \\
\hline Grass & A & Th & FD \\
\hline Grass & $\mathrm{P}$ & Th & NK \\
\hline Grass & $\mathrm{P}$ & $\mathrm{Th}$ & $\mathrm{AR}$ \\
\hline Grass & A & Th & FD \\
\hline
\end{tabular}




\begin{tabular}{|c|c|c|c|c|c|c|}
\hline & Cyperaceae & & & & & \\
\hline & Bulbostylis barbata (Roxb.) C.B.Cl. & & Sedge & A & Th & FD \\
\hline & Cyperus alopecuroides Rottb. Descr. & Hensuati & Sedge & $\mathrm{P}$ & Th & $\mathrm{AR}$ \\
\hline & $\begin{array}{l}\text { Cyperus brevifolius } \\
\text { (Rottb.)Hassk. }\end{array}$ & & Sedge & $\mathrm{P}$ & $\mathrm{Cr}$ & FD \\
\hline & Cyperus castaneus Willd. & & Sedge & A & Th & FD \\
\hline & Cyperus compressus $\mathrm{L}$. & & Sedge & A & Hel & FD \\
\hline & Cyperus difformis $\mathrm{L}$. & Swonli & Sedge & A & $\mathrm{Ch}$ & FD \\
\hline & Cyperus iria $\mathrm{L}$. & & Sedge & A & $\mathrm{Ch}$ & FD \\
\hline & Cyperus rotundus $\mathrm{L}$. & Mthaghas & Sedge & $\mathrm{P}$ & Hem & M \\
\hline & $\begin{array}{l}\text { Eleocharis acutangula (Roxb.) } \\
\text { Schult. \& Schult. }\end{array}$ & & Sedge & $\mathrm{P}$ & $\mathrm{Cr}$ & NK \\
\hline & $\begin{array}{l}\text { Eleocharis dulcis (Burm. f.) } \\
\text { Henschef }\end{array}$ & & Sedge & A & $\mathrm{Cr}$ & NK \\
\hline & Fimbristylis dichotoma (L.) Vahl & & Sedge & A & $\mathrm{Ch}$ & FD \\
\hline & Fimbristylis ferruginea (L.) Vahl & & Sedge & $\mathrm{P}$ & Hem & FD \\
\hline & Fimbristylis miliacea (L.) Vahl & & Sedge & A & Hem & FD \\
\hline & Fimbristylis ovata (Burm.f.) J Kern. & & Sedge & A & Hem & FD \\
\hline & Fuirena ciliaris (L.) Roxb. & & Sedge & A & Th & FD \\
\hline & $\begin{array}{l}\text { Kylinga nemoralis (J.R. \& G. Forst) } \\
\text { Dandy ex Hutch. \& Dalz. }\end{array}$ & & Sedge & $\mathrm{P}$ & Th & NK \\
\hline & Scirpus articulatus L. & Kanri & Sedge & A & Hem & NK \\
\hline & Scirpus grossus L. & Santara & Sedge & $\mathrm{P}$ & $\mathrm{Cr}$ & NK \\
\hline & Typhaceae & & & & & \\
\hline & Typha angustata Bory. \& Chaub & Hangla & Forb & $\mathrm{P}$ & $\mathrm{Ph}$ & M \\
\hline CORE EUDIC & & & & & & \\
\hline Proteales & Nelumbonaceae & & & & & \\
\hline & Nelumbo nucifera Gaertn. & Padma & Forb & $\mathrm{P}$ & Hyd & $\mathrm{E}$ \\
\hline Ranunculales & Menispermaceae & & & & & \\
\hline & Cissampelos pareira $\mathrm{L}$. & Akanbindi & Climber & $\mathrm{P}$ & $\mathrm{Ch}$ & M \\
\hline & Tiliacora racemosa Colebr. & Kalajati noi & Climber & $\mathrm{P}$ & $\mathrm{Ch}$ & NK \\
\hline & $\begin{array}{l}\text { Tinospora cordifolia (Willd.)Hook.f. } \\
\& \text { Thomson }\end{array}$ & Guluchilata & Climber & $\mathrm{P}$ & $\mathrm{Ch}$ & M \\
\hline & Papaveraceae & & & & & \\
\hline & Argemone mexicana $\mathrm{L}$. & Kantakusuma & Forb & A & Th & M,E \\
\hline ROSIDS & & & & & & \\
\hline Vitales & Vitaceae & & & & & \\
\hline & Cissus quadrangularis $\mathrm{L}$. & Hadabhanga & Shrub & $\mathrm{P}$ & Th & M \\
\hline FABIDS & & & & & & \\
\hline Zygophyllales & Zygophyllaceae & & & & & \\
\hline & Tribulus terrestris $\mathrm{L}$. & Gokhara & Forb & $\mathrm{P}$ & $\mathrm{Ch}$ & $\mathrm{M}$ \\
\hline Oxalidales & Oxalidaceae & & & & & \\
\hline & Oxalis corniculata $\mathrm{L}$. & Ambiliti & Forb & $\mathrm{P}$ & $\mathrm{Cr}$ & $\mathrm{M}, \mathrm{E}$ \\
\hline Malpighiales & Euphorbiaceae & & & & & \\
\hline & Acalypha indica $\mathrm{L}$. & & Forb & A & Th & NK \\
\hline & Chrozophora rottleri (Geisel.) Juss. & & Forb & A & Th & NK \\
\hline & Croton sparsiflorus Morong & Nandababuli & Forb & $\mathrm{P}$ & Th & $\mathrm{M}$ \\
\hline & Euphorbia hirta L. & Harharika & Forb & A & Th & M \\
\hline & Euphorbia heterophyla L. & & Forb & A & Th & NK \\
\hline & Euphorbia thymifolia L. & Patrasiju & Shrub & $\mathrm{P}$ & Th & $\mathrm{BF}$ \\
\hline & Euphorbia tirucalli L. & Dangulisiju & Shrub & $\mathrm{P}$ & Th & $\mathrm{BF}$ \\
\hline
\end{tabular}


Euphorbia tithymaloides L.

Jatropha curcas L.

Jatropha gossypiifolia L.

Synadenium grantii Hook $\mathrm{f}$.

Tragia involucrata $\mathrm{L}$.

LINACEAE

Linum usitatissimum $\mathrm{L}$.

\section{Phyllanthaceae}

Breynia vitis-idaea (Burm. f.)

Fischer

Phyllanthus amarus Schum.

\&Thonn.

Phyllanthus urinaria L.

Violaceae

Hybanthus enneaspermus (L.) F.

Muell.

Fabales

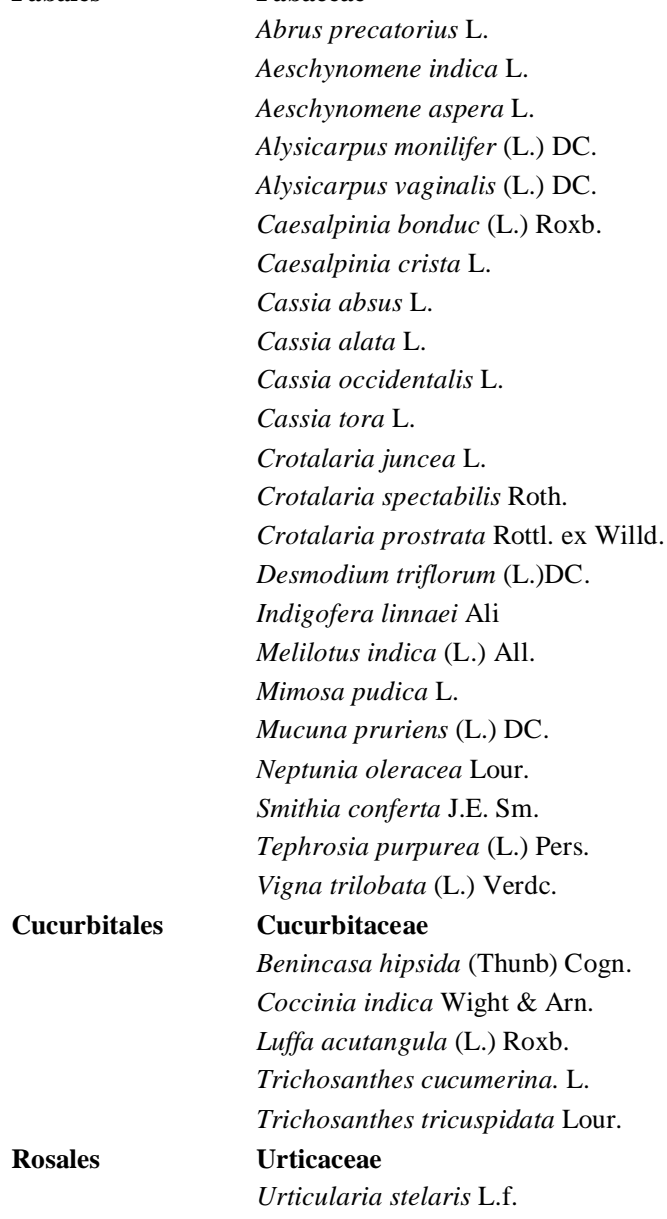

$\begin{array}{lllll} & \text { Shrub } & \text { P } & \text { Th } & \text { BF } \\ \text { Jara } & \text { Shrub } & \text { P } & \text { Ph } & \text { M } \\ \text { Baigaba } & \text { Shrub } & \text { P } & \text { Th } & \text { M } \\ & \text { Shrub } & \text { P } & \text { Th } & \text { BF } \\ \text { Bichhuati } & \text { Forb } & \text { A } & \text { Ph } & \text { M }\end{array}$

Bichsuati

$\begin{array}{lllll}\text { Pohalakuli Shrub } & \text { P } & \text { Th } & \text { BF }\end{array}$

Bhuianla Forb A Th M

Bhuiamla Forb A Th M

Madan mastak Forb A Ch M

Kaincha Legume $\mathrm{A} \quad \mathrm{Ph} \quad \mathrm{M}$

Sola Legume A $\quad$ Cr $\quad$ FD

Sola Legume A $\quad \mathrm{Cr} \quad \mathrm{AR}$

Legume A Th FD

Legume A Th FD

Gilo Climber $\mathrm{P} \quad \mathrm{Ph} \quad \mathrm{M}$

Nantei Climber $\mathrm{P} \quad \mathrm{Ph} \quad$ NK

Forb A Th NK

Shrub $\mathrm{P} \quad \mathrm{Ph} \quad \mathrm{M}$

Kalachakunda Forb $\quad \mathrm{P} \quad \mathrm{Ph} \quad \mathrm{M}$

$\begin{array}{lllll}\text { Chakunda } & \text { Forb } & \mathrm{P} & \text { Th } & \mathrm{M}\end{array}$

Chanapata Legume A Th M

Jhumka Legume $\mathrm{P}$ Th $\mathrm{FD}$

$\begin{array}{llll}\text { Jhumka Legume } & P & \text { Th } & \text { FD }\end{array}$

Kaansisna Legume A Th FD

Legume $\mathrm{P}$ Th FD

Bana methi Legume A Th FD

Lajakuli Legume $\mathrm{P}$ Th $\mathrm{M}$

Baidanka Climber A Th M

Legume A Hyd M

Sanomungo Legume A Th FD

Banakolathi Legume $\mathrm{P}$ Th $\mathrm{M}$

Legume A $\quad \mathrm{Cr} \quad \mathrm{FD}$

Panikakharu Climber A Ph E

Kunduri Climber A $\mathrm{Ph}$ E

Pitataradi Climber A $\quad \mathrm{Ph} \quad \mathrm{M}$

Banapotala Climber A Th E

Mahakal Climber P Th NK

Bhaturia dala Forb A Hyd NK 
MALVIDS

Myrtales

Brassicales

Sapindales

Malvales

Saxifragales

Caryophyllales

\section{Onagraceae}

Ludwigia adscendens (L.) H. Hara

Ludwigia hyssopifolia (G.Don)

Ludwigia octovalvis (Jacq.) Raven

Ludwigia perennis $\mathrm{L}$.

Lythraceae

Ammannia baccifera $\mathrm{L}$.

Ammannia multiflora Roxb.

Rotala indica (Willd.) Koehne

Jagal
Latkera
Ramdauni

Brassicaceae

Brassica juncea (L.) Czern. \& Coss.

Cleomaceae

Cleome gynandra $\mathrm{L}$.

Cleome monophylla $\mathrm{L}$.

Cleome rutidosperma DC.

Cleome viscosa $\mathrm{L}$.

Capparaceae

Capparis zeylanica $\mathrm{L}$.

Sapindaceae

Cardiospermum halicacabum L.

\section{Malvaceae}

Abutilon indicum (L.) Sweet

Corchorus aestuans L.

Corchorus olitorius $\mathrm{L}$.

Corchorus tridens $\mathrm{L}$.

Corchorus trilocularis $\mathrm{L}$.

Hibiscus sabdariffa $\mathrm{L}$.

Hibiscus vitifolius $\mathrm{L}$.

Malachra capitata (L.) L.

Malvaviscus arboreus Cav.

Melochia corchorifolia L.

Pavonia zeylanica (L.) Cav.

Sida acuta Burm.f.

Sida cordata (Burm. f.) Borss.

Waalk.

Sida cordifolia $\mathrm{L}$.

Sida rhombifolia $\mathrm{L}$.

Sida spinosa $\mathrm{L}$.

Triumfetta rhomboidea Jacq.

Urena lobata $\mathrm{L}$.

Crassulaceae

Bryophyllum pinnatum (Lam.) Oken.

\section{Aizoaceae}

Sesuvium portulacastrum (L.) L.

Trianthema portulacastrum $\mathrm{L}$.

Polygonaceae

Antigonon leptopus Hook. \& Arn.

Polygonum barbatum L.
Raisorisha

Arakasago

Rangasorish

Anasorisho

Kanphuta

Pedipedika

Bananalita

Khatakaunria

(ankamant

Telpuri

Sunakhadika

Bisiripi

Bisiripi

Sahabeda

Bajramuli

Amarpoi

Godabani

Purinisaga

Nara

$\begin{array}{llll}\text { Forb } & \text { A } & \text { Hyd } & \text { M,E } \\ \text { Forb } & \text { A } & \text { Hyd } & \text { M, E } \\ \text { Forb } & \text { A } & \text { Hyd } & \text { M } \\ \text { Forb } & \text { A } & \text { Hyd } & \text { FD } \\ & & & \\ \text { Forb } & \text { A } & \text { Th } & \text { FD } \\ \text { Forb } & \text { A } & \text { Th } & \text { FD } \\ \text { Forb } & \text { A } & \text { Th } & \text { FD }\end{array}$

Forb

A Th

E

Forb A Th M

Forb A Th NK

Forb A Th M

Forb A Th M

Shrub P Ph M

Climber A Th M

Shrub A Ph M

Forb A Th M

Forb A Th NK

Forb A Th NK

Forb A Th NK

Shrub A Th E

Shrub A Th NK

Forb P Th NK

Shrub $P$ Th NK

Forb A Th NK

Forb A Ch NK

Forb A Th M

Forb A Th M

Shrub A Th M

Shrub A Th M

Forb A Th FD

Shrub P Th NK

Shrub A Ph NK

Forb $\quad \mathrm{P} \quad \mathrm{Ch} \quad$ NK

Ferb $\quad P \quad$ Th $\quad$ BF

Forb A Th M

Climber $P \quad$ Th $\quad M$

Forb A Th E 


\begin{tabular}{|c|c|c|c|c|c|}
\hline Polygonum glabrum Willd & Bihongi & Forb & A & Th & E \\
\hline Polygonum plebeium R.Br. & Muthisaga & Forb & A & Th & $\mathrm{M}, \mathrm{E}$ \\
\hline \multicolumn{6}{|l|}{ Molluginaceae } \\
\hline Glinus oppositifolius (L.) A.DC. & Pitasaga & Forb & A & Th & $\mathrm{M}, \mathrm{E}$ \\
\hline \multicolumn{6}{|l|}{ Amaranthaceae } \\
\hline Achyranthes aspera $\mathrm{L}$. & Apamaranga & Forb & A & Th & M \\
\hline Aerva lanata (L.) Juss. ex Sch. & Paunsia & Forb & A & $\mathrm{Th}$ & FD \\
\hline $\begin{array}{l}\text { Alternanthera philoxeroides (Mart) } \\
\text { Griseb. }\end{array}$ & $\begin{array}{l}\text { Ghodamadarang } \\
\text { a }\end{array}$ & Forb & $\mathrm{P}$ & Hyd & FD \\
\hline $\begin{array}{l}\text { Alternanthera sessilis (L.)R.Br.ex } \\
\text { DC. }\end{array}$ & Madranga & Forb & $\mathrm{P}$ & Hyd & $\mathrm{M}, \mathrm{E}$ \\
\hline Amaranthus gangeticus L. & Nalikosala & Forb & A & Th & $\mathrm{E}$ \\
\hline Amaranthus viridis L. & Leutia & Forb & A & Th & $\mathrm{E}$ \\
\hline Amaranthus spinosus L. & Kantaneutia & Forb & A & Th & $\mathrm{M}, \mathrm{E}$ \\
\hline Celosia argentea $\mathrm{L}$. & Chulia & Forb & A & $\mathrm{Ch}$ & M \\
\hline Chenopodium album $\mathrm{L}$. & Bathuasaga & Forb & A & Th & $\mathrm{E}$ \\
\hline Gomphrena serrata L. & & Forb & A & Th & M \\
\hline Suaeda maritima (L.) Dumort. & & Forb & A & Th & NK \\
\hline \multicolumn{6}{|l|}{ Portulacaceae } \\
\hline Portulaca oleracea L. & Badabalbaula & Forb & A & $\mathrm{Ch}$ & $\mathrm{M}, \mathrm{E}$ \\
\hline Portulaca quadrifida $\mathrm{L}$. & Balbaula & Forb & A & $\mathrm{Ch}$ & E \\
\hline \multicolumn{6}{|l|}{ Nyctaginaceae } \\
\hline Boerhavia diffusa $\mathrm{L}$. & Puruni & Forb & $\mathrm{P}$ & Th & $\mathrm{M}, \mathrm{E}$ \\
\hline Mirabilis jalaba $\mathrm{L}$. & Chandrakanta & Forb & A & Th & M \\
\hline \multicolumn{6}{|l|}{ Cactaceae } \\
\hline $\begin{array}{l}\text { Opuntia stricta (Haw.) Haw. var. } \\
\text { dillenii (Ker Gawl.) L. D. Benson }\end{array}$ & Nagapheni & Shrub & $\mathrm{P}$ & $\mathrm{Ph}$ & $\mathrm{M}, \mathrm{BF}$ \\
\hline $\begin{array}{l}\text { Pilosocereus arrabidae } \\
\text { (Lem.) Byles \& G.D.Rowley }\end{array}$ & Deulisiju & Shrub & $\mathrm{P}$ & $\mathrm{Ph}$ & $\mathrm{BF}$ \\
\hline \multicolumn{6}{|l|}{ Plumbaginaceae } \\
\hline Plumbago zeylanica $\mathrm{L}$. & Chintamani & Forb & $\mathrm{P}$ & Th & M \\
\hline \multicolumn{6}{|l|}{ Apocynaceae } \\
\hline Calotropis gigantea $\mathrm{R} . \mathrm{Br}$. & Dhala-arakha & Shrub & $\mathrm{P}$ & $\mathrm{Ph}$ & M \\
\hline $\begin{array}{l}\text { Calotropis procera (Aiton)W.T. } \\
\text { Aiton }\end{array}$ & Arakha & Shrub & $\mathrm{P}$ & $\mathrm{Ph}$ & M \\
\hline Cathranthus roseus (L.) G. Don. & Sadabihari & Forb & $\mathrm{P}$ & Th & M \\
\hline $\begin{array}{l}\text { Gymnema sylvestre (Retz.) R.Br.ex } \\
\text { Schult. }\end{array}$ & Gurmari & Climber & $\mathrm{P}$ & Th & M \\
\hline $\begin{array}{l}\text { Pergularia daemia (Forssk.) Chiov. } \\
\text { Rubiaceae }\end{array}$ & Uturudi & Climber & $\mathrm{P}$ & Th & M \\
\hline Dentella repens (L.) J.R. \& Forst. & & Forb & A & Th & $\mathrm{BF}$ \\
\hline Hedyotis corymbosa $(\mathrm{L}$.$) Lam.$ & Jarjati & Forb & A & $\mathrm{Ch}$ & $\mathrm{M}$ \\
\hline Spermacoce articularis L.f. & Solaganthi & Forb & A & $\mathrm{Ch}$ & FD \\
\hline \multicolumn{6}{|l|}{ Acanthaceae } \\
\hline $\begin{array}{l}\text { Andrographis paniculata (Burm.f.) } \\
\text { Wall.ex. Nees }\end{array}$ & Bhuinnimba & Forb & A & Th & M \\
\hline Acanthus ilicifolius $\mathrm{L}$. & Harkanch & Shrub & $\mathrm{P}$ & Hel & M \\
\hline Barleria prionitis L. & Daskeraanta & Shrub & $\mathrm{P}$ & $\mathrm{Ch}$ & \\
\hline
\end{tabular}


Hygrophila auriculata Heine.
Justicia gendarussa Burn.f.
Ruellia prostrata Poir.
Rungia pectinata (L.) Nees
Lamiaceae
Clerodendrum inerme (L.) Gaertn.
Clerodendrum indicum (L.) Kuntze
Clerodendrum philippinum Schauer.
Clerodendrum phlomoides L. f.
Hyptis suaveolens (L.) Poit.
Leucas aspera (Willd.) Link
Ocimum canum Sims
Martyniaceae
Martynia annua L.
Scrophulariaceae
Bacopa monnieri (L.) Pennell
Lindernia antipoda (L.) Alston
Lindernia crustacea (L.)F.v.Muell.
Plantaginaceae

Mecardonia procumbens (Mills.)

Small

Scoparia dulcis L.

Pedaliaceae

Pedalium murex $\mathrm{L}$.

Sesamum indicum $\mathrm{L}$.

Verbenaceae

Duranta repens $\mathrm{L}$.

Lantana camara $\mathrm{L}$.

Lippia javanica (Burn.f) Spreng

Phyla nudiflora (L.) Greene

Solanales

\section{Convolvulaceae}

Argyreia nervosa (Burm. f.) Boj.

Cuscuta reflexa Roxb.

Evolvulus alsinoides (L.) L.

Evolvulus nummularius (L.) L.

Ipomoea alba $\mathrm{L}$.

Ipomoea aquatica Forssk.

Ipomoea carnea Jacq.

Ipomoea hederifolia $\mathrm{L}$.

Ipomoea marginata (Desr.) Verdc.

Ipomoea mauritiana Jacq.

Ipomoea obscura Ker.-Gawl.

Ipomoea pes-tigridis $\mathrm{L}$.

Ipomoea quamocit $\mathrm{L}$.

Merremia tridentata

(L.) Hall. f.

Merremia hederacea (Burm. f.)

Hall.

\begin{tabular}{|c|c|c|c|c|}
\hline Koelekha & Forb & $\mathrm{P}$ & $\mathrm{Ch}$ & M \\
\hline \multirow[t]{3}{*}{ Kalabasanga } & Forb & $\mathrm{P}$ & $\mathrm{Ch}$ & M \\
\hline & Forb & $\mathrm{P}$ & Th & NK \\
\hline & Forb & A & Th & NK \\
\hline Chinyanrhi & Shrub & $\mathrm{P}$ & $\mathrm{Ph}$ & M \\
\hline Nagri & Shrub & $\mathrm{P}$ & $\mathrm{Ph}$ & M \\
\hline Dilbari & Shrub & $\mathrm{P}$ & $\mathrm{Ph}$ & $\mathrm{BF}$ \\
\hline \multirow[t]{2}{*}{ Donkari } & Shrub & $\mathrm{P}$ & $\mathrm{Ph}$ & $\mathrm{M}$ \\
\hline & Forb & A & $\mathrm{Ph}$ & NK \\
\hline \multirow[t]{2}{*}{ Gaiso } & Forb & $\mathrm{P}$ & $\mathrm{Ch}$ & $\mathrm{M}$ \\
\hline & Forb & $\mathrm{P}$ & $\mathrm{Th}$ & M \\
\hline Baghanakhi & Shrub & A & $\mathrm{Th}$ & NK \\
\hline \multirow[t]{4}{*}{ Brahmi } & Forb & A & $\mathrm{Ch}$ & $\mathrm{M}, \mathrm{E}$ \\
\hline & Forb & $\mathrm{A}$ & Th & FD \\
\hline & Forb & $\mathrm{A}$ & $\mathrm{Th}$ & NK \\
\hline & Forb & A & $\mathrm{Ch}$ & NK \\
\hline Chirarita & Forb & $\mathrm{P}$ & $\mathrm{Ch}$ & NK \\
\hline Gokara & Forb & A & $\mathrm{Th}$ & M \\
\hline Khasa & Forb & A & $\mathrm{Ch}$ & M \\
\hline Bilatikanta & Shrub & $\mathrm{P}$ & $\mathrm{Ph}$ & $\mathrm{BF}$ \\
\hline Gandhagauria & Shrub & $\mathrm{P}$ & $\mathrm{Ph}$ & M \\
\hline \multirow[t]{2}{*}{ Naguari } & Forb & $\mathrm{P}$ & $\mathrm{Ch}$ & M \\
\hline & Forb & A & Th & FD \\
\hline Mundanoi & Climber & A & $\mathrm{Ph}$ & NK \\
\hline Nirmuli & Climber & A & Hem & $\mathrm{M}$ \\
\hline \multirow[t]{2}{*}{ Bichhamalia } & Forb & $\mathrm{P}$ & Th & $\mathrm{M}$ \\
\hline & Forb & $\mathrm{P}$ & Th & NK \\
\hline Kunjalata & Climber & $\mathrm{P}$ & Th & NK \\
\hline Kalamasaga & Climber & $\mathrm{A}$ & Hyd & $\mathrm{M}, \mathrm{E}$ \\
\hline \multirow[t]{3}{*}{ Amari } & Shrub & $\mathrm{P}$ & $\mathrm{Cr}$ & $\mathrm{BF}$ \\
\hline & Climber & $\mathrm{A}$ & $\mathrm{Th}$ & NK \\
\hline & Climber & $\mathrm{A}$ & Th & NK \\
\hline \multirow[t]{6}{*}{ Bhuinkakharu } & Climber & A & $\mathrm{Ph}$ & NK \\
\hline & Climber & $\mathrm{P}$ & Th & $\mathrm{M}$ \\
\hline & Climber & A & Th & NK \\
\hline & Climber & $\mathrm{P}$ & Th & $\mathrm{M}$ \\
\hline & Climber & $\mathrm{A}$ & $\mathrm{Th}$ & FD \\
\hline & Climber & A & Th & NK \\
\hline
\end{tabular}




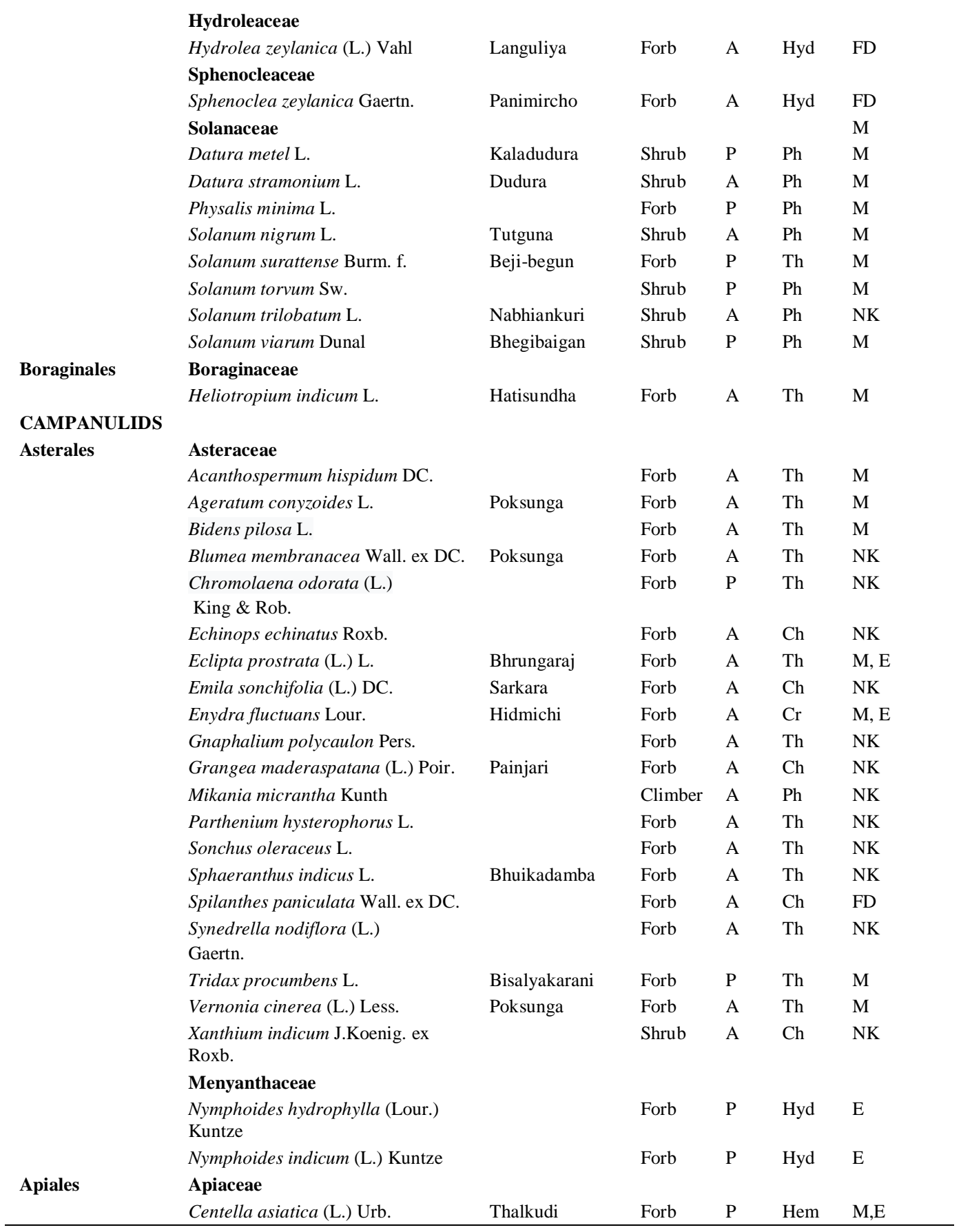

Abbreviations: A: Annual, P: Perennial, Ch: Chamephyte, Cr: Cryptophyte, Hem: Hemicryptophyte, Ph: Phanerophyte, Tel: Helophytes, Hyd: Hydrophyte, Th: Therophyte, M: Medicine, E: Edible, BF: Biofencing, FD: Fodder, R: Ritual, AR: Artifact, NK: Not known. 
was Poaceae represented by 29 species while the Fabaceae and Asteraceae were represented by 23 and 20 species, respectively. Habit analysis revealed that forbs were the most represented (50.5\%), followed by shrubs $(15.2 \%)$, climbers $(11.2 \%)$, grasses $(10.8 \%)$, sedges $(6.5 \%)$ and legumes (5.8\%). The Therophytes was the most dominant class with 135 plant species (48.7\%) followed by Phanerophytes with 40 species (14.5\%), Chemaephytes with 36 species (13.0\%), Hydrophytes with 28 species (10.1\%), Cryptophytes with 20 species (7.2\%), Hemicryptophytes with 15 species (5.4\%),and helophytes having three species (1.1\%) respectively. Annuals were the most represented $(57.7 \%)$ than the perennials $(42.3 \%)$.

Many weeds were used by local communities as food, fodder, traditional medicines and other purposes. Out of 277 , about $41 \%$ of the species were used for the treatment of various ailments, such as diabetes, gastrointestinal disorders, fever, gynaecology, cardiovascular disorders, skin diseases, rheumatism, and dental caries. Prominent among them were Andrographis paniculata, Bacopa monnieri, Catharanthus roseus, Centella asiatica, Glinus oppositifolius, Gymnema sylvestre, Ipomoea aquatica, Oxalis corniculata, Solanum surattense and Tridax procumbens. Many weeds (27.5\%) were collected by the farmers for domestic animal feed. Examples include Alternanthera philoxeroides, Echinochloa crusgalli, Hydrolea zeylanica, Polygonum glabrum and Spermacoce articularis.Similarly, $12.4 \%$ of the reported plant species were used for edible purposes, for instance Alternanthera sessilis, Colocasia esculenta, Glinus oppositifolius, Ipomoea aquatica and Oxalis corniculata. Weeds like Aeschynomene aspera, Cyperus alopecuroides, and Vetiveria zizanioides were used for various household purposes. Likewise, a variety of plant species were used for biofencing pupose. Examples include, Bougainvillea spectabilis, Clerodendrum inerme, Duranta repens, Euphorbia tirucalli, Ipomoea carnea, Jatropha curcas and Pandanus fascicularis. Some of the plants like Cynodon dactylon and Desmostachya bipinnata, were used for various rituals by the inhabitants of the district.

Many factors such as increasing atmospheric temperature and $\mathrm{CO}_{2}$ level, variation in rainfall pattern and climate change are regarded as important indicator of weed species distribution in a geographical area (Patterson et al., 1999; Rodenburg et al., 2011). For instance, Datura stramonium, which needs high temperature for profuse growth (Cavero et al., 1999) and Setaria viridis requires warmer conditions germinated later in the (August) season (Dekker, 2003), would become a more competitive candidate under the climate change scenarios. A recent study indicated that Setaria viridis would be a problematic weed in maize-based cropping systems elsewhere, through synchrony with maize emergence, which is probably due to stimulation by increased temperature (Peters and Gerowitt, 2014). Under such a scenario, the distribution and prevalence of weeds will be problematic in crop ecosystems.

The total number of weed species reported in the present study (277 species) is significantly higher compared with those found in Nalbari district, Assam, India (217 species; Bhattacharjya and Sarma, 2016), Sundargarh District, Odisha, India (174 species; Mallick et al., 2015), Spain (175 species; Cirujeda et al., 2011) but lower than the species reported from Central Europe (381 species; Lososova et al., 2008) and Greece (278 species; Damanakis, 1983).The botanical families with greater representation are the Poaceae, followed by Asteraceae and Fabaceae. The present report draws support from earlier studies (Radosevich and Holt, 1984; Pullaiah, 2015).The predominance of herbaceous plants (forbs 140 species; grasses 30 species and sedges 18 species) found in this study is also reported by Irwin et al. (2015).Present study revealed the predominance of annual weed species over the perennial ones which is similar to the conclusion of Bergmeier (2006). The dominant life forms in biological spectrum of a region indicate the phytoclimate of that region (Thakur, 2015). The present observation indicates a higher percentage of the Therophytes (49.1\%) which is in accordance with the studies of Bhattacharjya and Sarma (2016). 
In this study Boerhavia diffusa is found abundantly in cultivated fields, waste lands, roadsides, pathways and gardens. Low (1991) reported that, $B$. diffusa is found in dry sandy nature of soils. Abeywardana and Hettiarachchi (2001) concluded that $B$. diffusa is a common weed present in sandy areas, while Chopra (1969) stated that $B$. diffusa grows all over the warmer region up to $2000 \mathrm{~m}$ an altitudinal range of Himalaya and grows easily in fields following the rainy time of year and in wastelands. In the present study, Commelina benghalensis is present in abundance that exposed moderate moist condition. Kaul et al. (2002) stated that $C$. benghalensis requires moist soil condition for establishment and after establishment it can also survive dry condition. Achyranthes aspera is commonly found in the district. Smith (1981) reported that A. aspera grow from sea level up to $900 \mathrm{~m}$. Similarly, Chenopodium album is found mainly in crop fields. Glemnitz et al., (2000) reported Chenopodium album from agricultural fields throughout Europe independently of climatic conditions. Some weeds are causing great concern in many parts of this district. Ageratum conyzoides is expanding at an alarming rate, especially in agricultural fields, road sides and even gardens. The weed is harmful to native species and has become a problem in agroecosystems (Negi and Hajra, 2007). Eichornia crassipes is of most nuisance as it causes hindrance by choking all possible water bodies and reducing their utility (Cilliers, 1991). Similarly, Lantana camara is spreading fast all over the district due to its better competitive ability and allelopathic effect (Sundaram and Hiremath, 2012). Mikania micrantha which is a fast growing species, is covering the habitats of the district and suppressing the growth of agricultural crops as well as natural vegetation through competition and allelopathic effects (Huang et al., 2009). Parthenium hysterophorus, a dominant weed of the study area, especially in wastelands, roadsides, railway tracks and foot paths, is an aggressive colonizer spreading rapidly suppressing native herbaceous flora. This weed is reported to be allergenic causing respiratory problems, dermatitis and asthma (Raghubanshi et al., 2005).

A number of weeds reported from the study area (40.8\%) are used by local people in traditional medicines for their primary health care. For instance, burned root ashes of Achyranthes aspera $\mathrm{L}$. are applied topically to reduce the pain of the skin infected with worms as well as to expel the dead worms out. Warmed leaves of Amaranthus spinosus are applied locally to cure boils and burns. Fresh leaf paste of Argemone mexicana is applied topically to treat eczema. Decoction of whole plant of Boerhavia diffusa is used to treat leucorrhoea. Juice of Cynodon dactylon is used to stop nose bleeding. Latex of Euphorbia hirta is effective for healing of wounds. Glinus oppositifolius is used either in raw or cooked form to cure various types of skin disease like scabies, itches etc. Mimosa pudica roots are chewed for toothache. Rhizome paste of Nymphaea nouchali is administered to regulate menstruation. Tender twig of Phyllanthus amarus is used to cure dysentery. Leaf decoction of Tephrosia purpurea is prescribed to women against post natal complications. Decoction of Tridax procumbens leaf is applied topically on the boils, cuts, sores, wounds and eczema to promote healing. Crushed tuber powder of Cyperus rotundus is taken orally for jaundice. The present finding draws support from the studies of Panda et al. (2014) and Mishra (2017). A number of weeds such as Alternanthera sessilis, Bacopa monneieri, Centella asiatica, Boerhavia diffusa, Commelina benghalensis, Eclipta prostrata, Enydra fluctuans, and Hygrophila auriculata are reported to have both therapeutic and dietary functions and hence are used as medicinal food remedy. This overlap indicates the close relationship between health and food. Overlapping between food and medicines is quite well known in traditional societies (Mishra et al., 2011).

Even though agricultural scientists and extension officers recommends eradicatation of the weeds, $89 \%$ of the most widespread and aggressive weeds in the world are edible (Rapoport et al., 1995). Moreover, many of these species have a high nutritional value and medicinal properties (Duke, 1992). The consumption of weeds is a world-wide phenomenon that is noted as having an 
important role for human nutrition (Duke, 1992; Turner et al., 2011). In the study area, weeds like Amaranthus spinosus, Glinus oppositifolius, Ipomoea aquatica, Monochoria hastata, Nymphaea pubescens, Oxalis corniculata, Portulaca oleracea and Portulaca quadrifida are used for edible purposes by the local inhabitants. The weed species in the present study contained approximately $27.5 \%$ fodder plants which are supported by Marcelino et al. (2005). A good number of artifact items and household articles are prepared from Aeschynomene aspera, Cyperus alopecuroides and Vetiveria zizanioides by the artisans of the district. Similar observations have also been made in earlier studies (Tripathy et al., 2014).

This paper provides a comprehensive documentation of the weed diversity of Bhadrak district along with their socio-economic values.Most plant species of the study area are of considerable ecological and economic importance, useful as bioresources to wild fauna and human beings. In contrast, some species recorded from this area are considered to be troublesome as they are invasive and weedy with rapid distribution; and the natural vegetation will be replaced by weeds in few years. Bhadrak district, like other areas of India, is developing rapidly, and this development has the potential to put the natural ecosystem under stress through increased human activities such as modern farming (application of fertilizers, irrigation and chemical spray), housing, road construction, and overgrazing; and this would lead to the loss of native species. For these reasons, additional research should be conducted to evaluate the intrinsic ecological values of the local flora and to incorporate characteristics of species composition with ecological functions will provide a baseline for planning and proper conservation measures to safeguard phytodiversity which is facing ever growing biotic stress.

\section{References}

Abeywardana, N. and Hettiarachchi, J.K.N. 2001. Statistics on the national demand for medicinal plants. Woodl. Ave., Kohuwala.

Algandaby, M.M. and Salama, M.E. 2016. Management of the noxious weed; Medicago polymorpha L. via allelopathy of some medicinal plants from Taif region, Saudi Arabia. Saudi J. Biol. Sci. 25(7): 13391347.

APG, III. 2009. An update of the angiosperm phylogeny group classification for the orders and families of flowering plants. Bot. J. Linn. Soc. 161(2):105-121.

Barbara, D.B., Stephen, D.M. and Swanton, C.J. 2003. Weed ecology in natural and agricultural systems. CABI Publishing, Cambridge, USA.

Bastiaans, L., Kropff, M.J., Goudriaanb, J. and Van Laar, H.H. 2000. Design of weed management systems with a reduced reliance on herbicides poses new challenges and prerequisites for modeling crop-weed interactions. Field Crop Res. 67: 161-179.

Bergmeier, E. 2006. The diversity of segetal weeds in Crete (Greece) at species and community level. Annali Di Botanica VI: 53-64.

Bhandari, D.C. and Sen, D.N. 1979. Agroecosystem analysis of the Indian arid zone. I. Indigofera cordiflora as a weed. Agro-Ecosystems5:257.

Bhattacharjya, D.K.and Sarma, S.K. 2016. Floristic composition and biological spectrum of weeds in agroclimatic zone of Nalbari district, Assam, India. Trop. Pl. Res. 3(3): 573-585

Cavero, J., Zaragoza, C., Suso, M.L. and Pard, A. 1999. Competition between maize and Datura stramonium in an irrigated field under semi-arid conditions. Weed Res. 39: 225-240.

Champion, H.G. and Seth, S.K. 1968. A revised survey of the forest types of India. Manager of Publications, New Delhi, India, pp.16-17.

Chopra, G.L. 1969. Angiosperms. Systematics and Life Cycle. S. Nagin \& Co. Jalandhar, Punjab, India, pp. 361-365. 
Cilliers, C.J. 1991. Biological control of water hyacinth, Eichhornia crassipes (Pontederiaceae), in South Africa. Agric. Ecosyst. Environ. 37: 207-218.

Cirujeda, A., Aibar, J. and Zaragoza, C. 2011. Remarkable changes of weed species in spanish cereal fields from 1976 to 2007. Agron. Sustain. Dev. 31(4): 675-688.

Crawley, M.J. 1997b. Biodiversity. In: Crawley, M.J. (Ed.), Plant Ecology. 2nd edn, Blackwell Scientific, Oxford, pp. 595-632.

Cunningham, A.B. 2001. Applied ethnobotany: People, wild plant use and conservation. Earthscan Publishing Ltd. London, UK.

Cunningham, S.C., Mac Nally, R., Baker, P.J., Cavagnaro, T.R., Beringer, J., Thomson, J.R. and Thompson, R.M. 2015. Balancing the environmental benefits ofreforestation in agricultural regions. Perspect. Plant Ecol. Evol. Syst. 17:301-317.

Damanakis, M. 1983. Weed species in wheat fields of Greece -1982, 1983 survey. Zizaniology 1: 85-90

Dekker, J. 2003. Evolutionary biology of the Foxtail (Setaria) species-group.In: Inderjit (Ed.), Weed Biology and Management.Kluwer Academic Publishers, Dordrecht, pp. 65-114.

DeWet, J.M.J. and Harlan, J.R. 1975. Weeds and domesticates: Evolution in the man-made habitat, Econ. Bot.29: 99-107.

Duke, J.A. 1992. Handbook of edible weeds. Boca Raton, Florida: CRC Press.

El-Sheikh, M.A. 2013a. Weed vegetation ecology of arable land in Salalah, Southern Oman. Saudi J. Biol. Sci. 20: 291-304.

Fried, G., Petit, S. and Reboud,X. 2010. A specialist-generalist classification of the arable flora and its response to changes in agricultural practices. BMC Ecol. 10(1): 20.

Gajbhiye, K.S. and Mandal, C. 2006: Agro-Ecological Zones, their Soil Resource and Cropping Systems. In: Status of farm mechanization in India, Indian Agri. Statistics Res. Institute, New Delhi, India, pp.1-32.

Glemnitz, M., Czimber, G., Radics, L. and Hoffmann, J. 2000. Weed flora composition along a north-south climate gradient in Europe. Acta Agrono'mica O 'va'riensis 42: 155-169.

Holm, L.G., Doll, E., Holm, J., Pancho, J.V. and Herberger, J.P. 1997. World Weeds: Natural Histories and Distribution. John Wiley \& Sons, Inc., New York.

Huang, Q.Q., Wu, J.M., Bai, Y.Y., Zhou, L. and Wang, G.X. 2009. Identifying the most noxious invasive plants in China: role of geographical origin, life form and means of introduction. Biodiver. Conser. 18: $305-316$.

Hyvonen, T. 2007: Can conversion to organic farming restore the species composition of arable weed communities. Biol. Conser.137:382-390.

Irwin, S.J., Thomas,S., Rathinaraj, P. and Duvuru, N. 2015. Angiosperm diversity of the Theosophical Society campus, Chennai, Tamil Nadu, India. Check List 11(2): 1579.

Kang, M.S. and Banga, S.S. 2013. Global agriculture and climate change: a perspective.In: Kang, M. S. and Banga, S.S. (Eds.), Combating Climate Change: An Agricultural Perspective.CRC Press, Boca Raton, pp. 11-25.

Kaul, V., N. Sharma, N. and Koul, A.K. 2002. Reproductive effort and sex allocation strategy in Commelina benghalensis L., a common monsoon weed. Bot. J. Linn. Soc. 140: 403-413.

Lososova, Z., Chytry, M. and Kuhn, I. 2008. Plant attributes determining the regional abundance of weeds on central European arable land. J. Biogeogra. 35: 177-187

Low, T. 1991. Wild Food Plants of Australia.North Ryde, N.S.W. Angus \& Robertson, Sydney.

Mallick, S.N., Maharana, M.R. and Acharya, B.C. 2015. Weed flora of Rourkela and adjoining areas of Sundargarh district, Odisha, India. J. Econ. Taxon. Bot. 39(1): 130-137.

Marcelino, L.R., Inocencio, A.I., Zaballa, C.C. and Paller, E.C. 2005: Bicol's weed recipes. Philipp. J. Weed Sci. 23: $40-43$.

Martin-Fores I., Guerin, G.R. and Lowe, A.J. 2017. Weed abundance is positively correlated with native plant diversity in grasslands of southern Australia. PLoS ONE 12(6): e0178681.

Martin, G.J. 1995. Ethnobotany:A methods manual.Chapman and Hall, London, UK. 
Mishra, N. 2017. Ethnomedicinal uses of some bioresources for healing of different ailments in Odisha,India. Ph. D. Thesis, V.K.S. University, Ara.

Mishra, N., Rout, S.D. and Panda,T. 2011. Ethnozoology and medicinal values of Similpal Biosphere Reserve, Orissa, India. Afri. J. Pharm.Pharmacog. 5(1): 6-11.

Navas, M. L. 1991. Using plant populations biology in weed research: A strategy to improve weed management. Weed Res. 31:171-179.

Negi, P.S. and Hajra, P.K. 2007. Alien flora of Doon Valley, North West Himalaya. Curr. Sci. 92(7): 968978.

Nyarko, K.A. and Datta, S.K.D.1993. Effects of light and nitrogen and their interaction on the dynamics of rice-weed competition. Weed Res. 33: 1-8.

Panda, D., Pradhan, S., Palita, S.K., Jayant, K. and Nayak, J.K. 2014. Medicinal weed diversity and ethno medicinal weeds used by tribal's of Koraput, India. Ecol. Environ. Conser. 20 (Suppl.): 35-38.

Patterson, D.T., Westbrook, J.K., Joyce, R.J.V., Lingren, P.D. and Rogasik, J. 1999. Weeds, insects, and diseases. Climate Change 43: 711-727.

Peters, K. and Gerowitt, B. 2014. Important maize weeds profit in growth and reproduction from climate change conditions represented by higher temperatures and reduced humidity. J. Appl. Bot. Food Qual. 87: $234-242$.

Pullaiah, T. 2015. Flora of Telangana - The 29th state of India. J. Indian Bot. Soc. 94(1\&2): 1-8.

Radosevich, S.R. and Holt, J.S. 1984. Weed Ecology: Implicationsn for Vegetation Management. John Wiley \& Sons, Inc., New York, USA.

Raghubanshi, A.S., Rai, L.C., Gaur, J.P. and Singh,J.S.2005. Invasive alien species and biodiversity in India. Curr.Sci.88(4):539-540.

Rao, A.N., Wani, S.P. and Ladha, J.K. 2014. Weed management research in India - an analysis of the past and outlook for future In: Souvenir (1989-2014). DWR Publication No. 18. Directorate of Weed Research, Jabalpur, India, pp. 1 -26.

Rapoport, E.H., Raffaele, E., Ghermandi, L. and Margutti, I.L. 1995. Edible weeds: a scarcely used resource. Bull. Ecol. Soc. Am.76(3):163-166.

Raunkiaer, C. 1934. The life forms of plants and statistical plant geography. Oxford University Press, Oxford.

Rejmanek, M. 1995. What makes a species invasive? In: Pysek, P., Prach, K., Rejmanek, M. and Wade, M.(Eds.), Plant Invasions - General Aspects and Specific Problems. SPB Academic Publishing, Amsterdam, pp. 3-13.

Rodenburg, J., Meinke, H. and Johnson, D.E. 2011. Challenges for weed management in African rice systems in a changing climate. J. Agric. Sci. 149: 427-435.

Saxena, H.O. and Brahmam, M.1996. The Flora of Orissa. Vol. I-IV, Orissa Forest Development Corporation, Bhubaneswar.

Singh, R.P., Singh, R.K. \& Singh, M.K. 2011. Impact of climate and carbon dioxide change on weeds and their management-A Review. Indian J. Weed Sci. 43(1\&2): 1-11.

Sinha, M.K. and Banerjee, A. 2018.Ecophysiological characteristics of selected dominant weeds of Sarguja division Chhattisgarh (India). Int. J. Biol. Res. 3(1): 91-97.

Smith, A.C. 1981. Flora Vitiensis Nova 2. Angiospermae Dicotyledones, Families. pp. 44-116.

Sundaram, B. and Hiremath, A.J. 2012. Lantana camara invasion in a heterogeneous landscape: patterns of spread and correlation with changes in native vegetation. Biol. Invasion14: 1127-1141.

Thakur, A.S. 2015. Floristic composition, life-forms and biological spectrum of tropical dry deciduous forest in Sagar District, Madhya Pradesh, India. Trop. Pl. Res. 2 (2): 112-119.

Thomas, J.M., Stephen, C.W. and Floyd, M.A. 2002. Weed sciences, principles and practices. John Wiley \& Sons, Inc., New York.

Tiwari, D., Upadhyay, S.S. and Paliwal, A.A. 2016.Diversity of weed flora of Bharsar, Pauri Garhwal (Uttarakhand), India.IOSR J.Agric. Vet. Sci. 9(11): 1-9. 
Travlos, I.S., Cheimona, N., Roussis, I. and Bilalis, D.J. 2018. Weed-species abundance and diversity indices in relation to tillage systems and fertilization. Front. Environ. Sci. 6:11.

Tripathy, B.K., Panda, T and Mohanty, R.B. 2014.Traditional artifacts from 'Bena' grass (Vetiveria zizanioides (L.) Nash., Poaceae) in Jajpur district of Odisha, India. Indian J. Trad. Know. 12(4): 771-777.

Turner, N.J., Łuczaj, L.J., Migliorini,P., Pieroni, A., Dreon, A.L. and Sacchetti,L.E. 2011. Edible and tended wild plants, traditional ecological knowledge and agroecology. Critical Rev. Pl. Sci.30 (1\&2):198-225.

Waterhouse, B.M. 2003. Know your enemy: recent records of potentially serious weeds in northern Australia, Papua New Guinea and Papua (Indonesia). Telopea 10(1): 477-485.

Wisler, G.C. and Norris, N.E. 2005. Interactions between weeds and cultivated plants as related to management of plant pathogens. Weed Sci. 53: 914-917.

Yamaguchi, H. and Umemoto, S. 1996. Classification of paddy levees in terms of plant resource complex. Weed Res. Japan 41: 286-294. (in Japanese with English summary)

(Manuscript received on 19 August 2019; revised on 18 May 2020) 